\title{
Outcomes following pharyngeal reconstruction in total laryngectomy - Institutional experience and review of literature
}

\author{
Deepak Balasubramanian', Narayana Subramaniam¹, Priyank Rathod', Samskruthi Murthy', \\ Mohit Sharma², Jimmy Mathew², Krishnakumar Thankappan', Subramania lyer ${ }^{1,2}$ \\ Departments of ${ }^{1} \mathrm{Head}$ and Neck Oncology and ${ }^{2}$ Plastic and Reconstructive Surgery, Amrita Hospital, Kochi, \\ Kerala, India
}

Address for correspondence: Dr. Deepak Balasubramanian, Department of Head and Neck Surgery and Oncology, Amrita Hospital, Ponnekkara Po, Kochi - 682 041, Kerala, India. E-mail: deepakbala@live.com

\section{ABSTRACT}

Background: Pharyngeal reconstruction is a challenging aspect of reconstruction after resections for head-and-neck cancer. The goals of reconstruction are to restore the continuity of the pharyngeal passage to enable oral alimentation and rehabilitation of speech wherever possible. This study was performed to determine the outcomes following pharyngeal reconstruction in total laryngectomy $(T L)$ using different reconstructive options and to determine the predictors of pharyngocutaneous fistula (PCF) and swallowing dysfunction. Materials and Methods: Retrospective analysis of patient data between 2003 and 2010 of patients undergoing TL with partial or total pharyngectomy. Demographic and treatment details were collected and analysed. Univariate analysis was performed to determine predictors of PCF and swallowing dysfunction. Results: Fifty-seven patients underwent pharyngeal reconstruction following TL, 31 of whom had received prior treatment. Following tumour resection, 31 patients had circumferential defects and 26 patients had partial pharyngeal defects. The flaps used include pectoralis major myocutaneous flap $(n=29)$, anterolateral thigh flap $(n=8)$, gastric pull-up $(n=13)$ and free jejunal flap $(n=7)$. PCF was seen in 20 patients, of which $15(75 \%)$ were managed conservatively and 5 required another surgery. At last follow-up, 99 patients $(68 \%)$ were on full oral alimentation. Tracheo-oesophageal puncture and prosthesis insertion was done in 20 patients, of whom $17(85 \%)$ developed satisfactory speech. Partial pharyngeal defects were associated with a higher risk of PCF on univariate analysis $(P=0.006)$ but were not significant on multivariate analysis. Post-operative swallowing dysfunction was significantly higher with hypopharyngeal involvement by tumour $(P=0.003)$. Conclusion: Pharyngeal reconstruction

\begin{tabular}{|l|l|}
\hline \multicolumn{2}{|c|}{ Access this article online } \\
\hline Quick Response Code: & Website: \\
\hline & www.ijps.org \\
\cline { 2 - 2 } & Dol: \\
\hline
\end{tabular}

This is an open access journal, and articles are distributed under the terms of the Creative Commons Attribution-NonCommercial-ShareAlike 4.0 License, which allows others to remix, tweak, and build upon the work non-commercially, as long as appropriate credit is given and the new creations are licensed under the identical terms.

For reprints contact: reprints@medknow.com

How to cite this article: Balasubramanian D, Subramaniam N, Rathod P, Murthy S, Sharma M, Mathew J, et al. Outcomes following pharyngeal reconstruction in total laryngectomy institutional experience and review of literature. Indian J Plast Surg 2018;51:190-5. 
in TL is feasible with good results. Majority of the patients swallow and regain acceptable swallowing function within 3 months.

\section{KEY WORDS}

Laryngectomy; pharyngeal reconstruction; pharyngocutaneous fistula

\section{INTRODUCTION}

dvanced laryngeal and hypopharyngeal cancer often require a TL as part of their multimodal treatment. In certain instances, a part or whole of the pharynx will need to be resected to ensure oncological margins. In the era of organ preservation chemoradiotherapy, the surgeon is often faced with salvage resections with its attendant complications. Pharyngeal reconstruction is required to enable oral feeding and voice rehabilitation with a tracheo-oesophageal puncture. This study was performed to determine the outcomes following pharyngeal reconstruction in TL and to determine the predictors of pharyngocutaneous fistula (PCF) and swallowing dysfunction.

\section{MATERIALS AND METHODS}

From a prospectively maintained database of patients treated at Amrita Institute of Medical Sciences, Kochi, we identified 57 patients who underwent pharyngeal reconstruction following TL between 2003 and 2010 . Information extracted from this database included patient demographics, prior treatment history, tumour details, surgical details, post-operative course of events, details of radiotherapy and follow-up details. The outcomes regarding speech, swallowing, fistula and stricture were noted.

\section{Statistical analysis}

Descriptive variables were summarised. Univariate analysis was performed using Pearson bivariate correlation coefficient, to assess determinants of fistula and dysphagia. Multivariate analysis was performed with logistic regression analysis. $P<0.05$ was considered statistically significant. Statistical analysis was done using SPSS version 22 (IBM Corp, Armonk, NY).

\section{RESULTS}

\section{Patient characteristics}

A total of $57(\mathrm{M}=44$ and $\mathrm{F}=13)$ patients underwent pharyngeal reconstruction following TL. The median age of patients was 55 years (range $42-72$ years). The median follow-up was 22 months (range 6-60 months). Patient and treatment characteristics are shown in Table 1.

\section{Tumour and prior treatment characteristics}

The sites involved by tumour included hypopharynx in 29 patients, larynx in 22 patients, oropharynx (posterior pharyngeal wall extending to the larynx) in 5 patients and thyroid gland with extensive laryngeal involvement in 1 patient.

Thirty-one patients (54\%) had prior treatment; chemoradiotherapy in 10 patients, radiotherapy in 9 patients, surgery with adjuvant chemoradiotherapy in 7 patients, surgery with adjuvant radiotherapy in 4 patients and induction chemotherapy in 1 patient. Those who received previous radiotherapy or

Table 1: Patient and treatment characteristics

\begin{tabular}{lc}
\hline Characteristics & Number of patients (\%) \\
\hline Age (years) & \\
$\geq 60$ & $22(39)$ \\
$<60$ & $35(61)$ \\
Sex & \\
Male & $44(77)$ \\
Female & $13(23)$ \\
Site involved & \\
Hypopharynx & $29(51)$ \\
Larynx & $22(39)$ \\
Oropharynx & $5(9)$ \\
Thyroid & $1(1)$ \\
Prior treatment & \\
None & $26(46)$ \\
Chemoradiotherapy & $10(18)$ \\
Radiotherapy & $9(16)$ \\
Surgery + chemoradiotherapy & $7(12)$ \\
Surgery + radiotherapy & $4(7)$ \\
Induction chemotherapy & $1(1)$ \\
Defect & \\
Circumferential & $31(54)$ \\
Partial & $26(46)$ \\
Flap used & \\
PMMC patch & $24(42)$ \\
PMMC tubed & $5(9)$ \\
ALT patch & $6(10)$ \\
ALT tubed & $2(4)$ \\
Gastric pull-up & $13(23)$ \\
Jejunal-free flap & $7(12)$ \\
\hline PMMC: Pectoralis major myocutaneous flap, ALT: Anterolateral thigh-free flap
\end{tabular}

Indian Journal of Plastic Surgery Volume 51 Issue 2 May-August 2018 
chemoradiotherapy had recurrent or residual disease after treatment requiring surgery, while those who previously underwent $\mathrm{TL}$ with primary closure and developed pharyngeal recurrences.

\section{Surgical technique employed}

All patients underwent a standard technique of closure. In patients in whom a cutaneous flap was used, an interrupted suturing in two layers with 3-0 vicryl was employed. The first layer of closure was a dermis-to-dermis approximation and the second layer was a muscle-to-muscle or fascia-to-fascia approximation of the flap. Proximally, the flap was spatulated, while distally a lock-and-key figure was used for approximation. In the cases of visceral flaps, the flap was sutured to the end of the defect with interrupted prolene sutures.

\section{Nature of defect and choice of flap}

Following tumour resection, 31 patients (54\%) had circumferential pharyngeal defects and 26 patients $(46 \%)$ had partial pharyngeal defects. The flaps used in our series included pectoralis major myocutaneous (PMMC) patch in 24 patients $(42 \%)$, tubed PMMC flap in 5 patients $(9 \%)$ [Figures 1-3], tubed anterolateral thigh (ALT)-free flap in 6 patients (11\%) and ALT-free flap patch in 2 patients (5\%), gastric pull-up in 12 patients (21\%) and free jejunal flap in 7 patients (12\%) [Figure 1].

Our protocol for pharyngeal reconstruction is shown in Figure 3. For patients having a patch defect, the PMMC flap is preferred in a vessel-depleted neck or in a salvage setting post-radiotherapy, where the vascularised muscle bulk has been shown to prevent PCF.[1] Otherwise, a radial forearm-free flap is used as it is thin and pliable, allowing deglutition. When mucosal and skin defects are present,

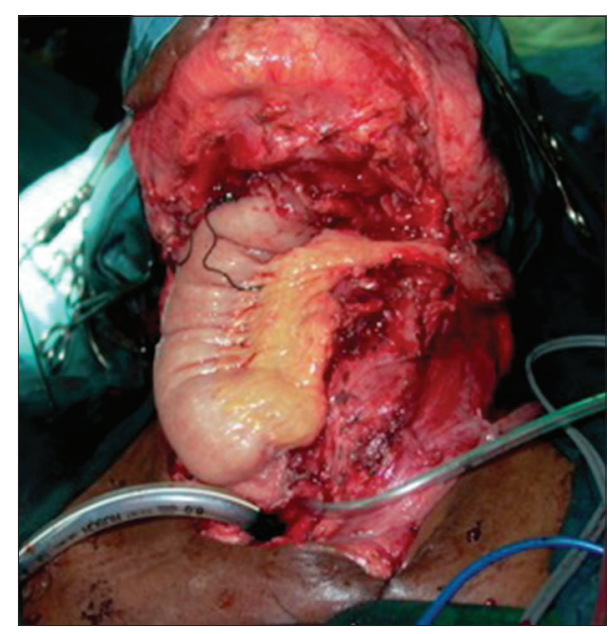

Figure 1: Pharyngeal reconstruction performed with jejunal-free flap the PMMC is used for similar indications as for patch defects, with the external surface of the flap covered with a split skin graft. When free flap reconstructions are suitable, depending on the patient's habitus, a radial forearm free flap or anterolateral thigh free flap are used; the goal is to have a thin pliable conduit. For circumferential defects where the lower end is accessible in the neck for anastomoses, PMMC is used again in salvage settings, in a vessel-depleted neck or in patients unfit for prolonged anaesthesia. The ALT is another suitable option for circumferential reconstruction if the thigh skin is not too thick or hairy; in either of these situations, a radial forearm is used. The jejunal-free flap can also be used; enteric flaps are associated with better swallowing outcomes than skin-lined flaps ${ }^{[2]}$ however, their harvest requires expertise and their tolerance to ischaemia is very limited and hence, considerable microsurgical expertise needs to be acquired before attempting them. For circumferential defects where the lower end is not easily accessible in the neck for a tension-free anastomosis, a gastric pull-up is preferred. In our institution, the jejunal flap is the choice for patients with circumferential defects with good surgical fitness, while ALT is the choice for patients who are less fit, in whom the additional morbidity of a laparoscopic harvest should be avoided. Gastric pull-up is used only when the lower resection stump is not available for anastomosis in the neck.

\section{Pharyngocutaneous fistula}

Post-operative PCF was seen in 20 patients (37\%), of which 15 of these patients were managed conservatively and 5 required surgical intervention in the form of a second flap reconstruction (PMMC flap in three and radial forearm-free flap in two).

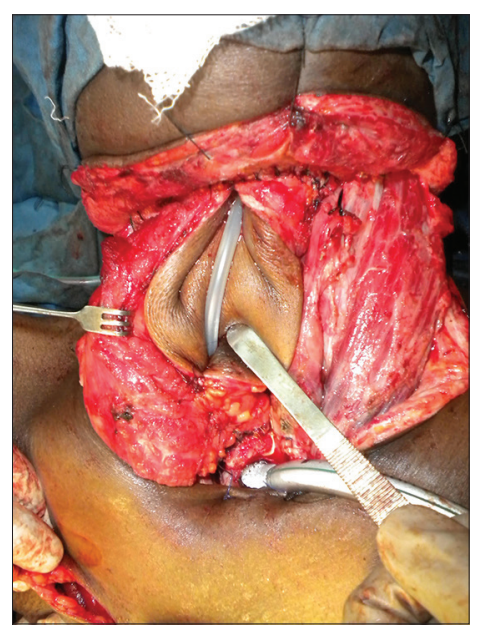

Figure 2: Pharyngeal reconstruction with a pectoralis major myocutaneous flap being tubed 


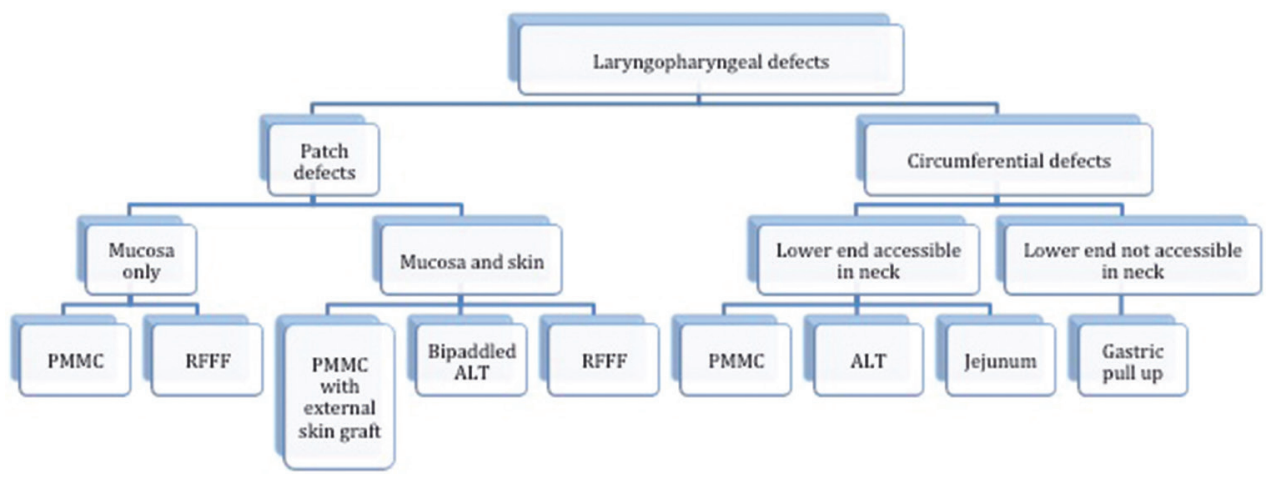

Figure 3: Our institutional algorithm for laryngopharyngeal reconstruction. PMMC: Pectoralis major myocutaneous flap, RFFF: Radial forearm-free flap, ALT: Anterolateral thigh flap

Our protocol for assessment of pharyngeal integrity after reconstruction was videofluoroscopy with a modified barium swallow on the $14^{\text {th }}$ post-operative day. Those without leaks were started on oral alimentation, while those with leaks were continued on nasogastric alimentation. For patients with minor leaks, conservative management was administered with nasogastric alimentation, high protein diet and wound care, with reassessment after 2 weeks. For those with significant leaks or those with more than 1 month of conservative management after the initial diagnosis of PCF, surgical correction was performed. We were unable to identify any predictors of persistent PCF requiring surgical correction.

The most common site of leak was in between the suture line $(80 \%)$ with the remaining occurring at either the proximal or distal circumferential suture line in tubed repairs (20\%). Complications following pharyngeal reconstruction are shown in Table 2, with the leak rate of individual flaps as shown.

When we performed a univariate analysis for the determinants of PCF [Figure 3], the only significant predictor of fistula was the presence of a partial pharyngeal defect; those with partial pharyngeal defects were associated with a fistula in $54 \%$, while those with circumferential defects were associated with a fistula in $31 \%$. However, on multivariate analysis, this was not significant; it is likely to have been confounded by factors like choice of flap and patient factors that could not be adequately adjusted for.

\section{Swallowing dysfunction}

Full oral alimentation was restored by the $1^{\text {st }}$ month in 7 , in the $2^{\text {nd }}$ month by 13 patients and in the $3^{\text {rd }}$ month by nine patients; $40 \%$ of patients were on full oral alimentation
Table 2: Complications following pharyngeal reconstruction

\begin{tabular}{lc}
\hline Complications & Number of patients (\%) \\
\hline Fistula & \\
PMMC patch & $12 / 24(50)$ \\
PMMC tubed & $3 / 5(60)$ \\
ALT patch & $1 / 2(50)$ \\
ALT tube & $1 / 6(16)$ \\
Gastric pull-up & $2 / 12(16)$ \\
Jejunal-free flap & $1 / 6(16)$ \\
Pharyngeal stricture & \\
PMMC patch & $4 / 24(16)$ \\
PMMC tubed & 0 \\
ALT patch & 0 \\
ALT tubed & 0 \\
Gastric pull-up & 0 \\
Jejunal-free flap & $4 / 6(66)$ \\
Swallowing dysfunction & \\
PMMC patch & $5 / 24(21)$ \\
PMMC tubed & $1 / 5(20)$ \\
ALT patch & $1 / 2(50)$ \\
ALT tubed & $4 / 6(66)$ \\
Gastric pull-up & $4 / 12(33)$ \\
Jejunal-free flap & $1 / 6(16)$ \\
\hline PMMC: Pectoralis major myocutaneous flap, ALT: Anterolateral thigh-free flap
\end{tabular}

within 3 months after surgery. Another ten patients (18\%) had oral alimentation restored between 6 and 12 months following surgery.

The reasons for dysphagia in these patients were strictures, the presence of pseudodiverticuli or reduced propulsion in the neopharynx. Eight patients (15\%) developed pharyngeal strictures of which five were dilated successfully. The remaining patients underwent a feeding procedure in the form of a percutaneous endoscopic gastrostomy or feeding jejunostomy.

At last follow-up, 99 patients $(72 \%)$ were on full oral alimentation. On univariate analysis, the only predictor of poor swallowing was hypopharyngeal involvement by tumour (pharynx vs. larynx, $P=0.046$ ), as shown in 
Table 3. None of the characteristics, such as age and the nature of defect or the nature of flap used, were found to impact post-operative dysphagia [Table 4].

\section{Speech outcomes}

Tracheo-oesophageal puncture and prosthesis insertion were done in 20 patients (35\%). Those with enteric flaps or persistent PCF did not undergo prosthesis placement in the primary setting. Of these 20 patients, 17 (85\%) developed satisfactory speech. Of those who did not receive tracheo-oesophageal punctures, rehabilitation was offered with electrolarynx.

\section{Mortality}

Three patients $(5 \%)$ died in the post-operative period. Of these, one was due to acute coronary syndrome in

Table 3: Patient characteristics associated with fistula

\begin{tabular}{|c|c|c|c|c|}
\hline Characteristic & $\begin{array}{c}\text { Without } \\
\text { fistula }\end{array}$ & $\begin{array}{c}\text { With } \\
\text { fistula }\end{array}$ & $\begin{array}{c}P \text { value on } \\
\text { univariate } \\
\text { analysis }\end{array}$ & $\begin{array}{l}P \text { value on } \\
\text { multivariate } \\
\text { analysis }\end{array}$ \\
\hline \multicolumn{5}{|l|}{ Age (years) } \\
\hline$<60$ & 23 & 12 & 0.872 & - \\
\hline$\geq 60$ & 14 & 8 & & \\
\hline \multicolumn{5}{|l|}{ Sex } \\
\hline Male & 27 & 17 & 0.302 & - \\
\hline Female & 10 & 3 & & \\
\hline \multicolumn{5}{|l|}{ Site } \\
\hline Pharyngeal & 21 & 13 & 0.624 & - \\
\hline Laryngeal & 15 & 7 & & \\
\hline \multicolumn{5}{|l|}{ Pharyngeal defect } \\
\hline Circumferential & 24 & 7 & $0.006^{*}$ & Not significant \\
\hline Partial & 9 & 13 & & \\
\hline \multicolumn{5}{|l|}{ Flap used } \\
\hline Regional & 26 & 16 & 0.425 & - \\
\hline Free & 11 & 4 & & \\
\hline
\end{tabular}

the perioperative period and two were following PCF, leading to sepsis. Of the 20 patients developing PCF, the mortality rate was $10 \%$.

\section{DISCUSSION}

Pharyngeal reconstruction remains a continuous challenge post-ablation for head-and-neck cancer. With the increasing use of organ preservation protocols in the treatment of laryngeal and hypopharyngeal cancers, surgery is often performed in a salvage setting. These results often results in extensive defects and significant local toxicity resulting in a higher local complication rate following surgery.

A large series that those receiving previous chemoradiotherapy have a significantly higher incidence of wound complications (45\% vs. 25\%) and PCF rate $(32 \%$ vs. $12 \%$ ) when compared to those undergoing surgery without any previous therapy. ${ }^{[3]}$ This was reflected in our cohort of patients, with over half of the patients having received previous treatment and a PCF rate of around $37 \%$. However, when we analysed for determinants of fistula, the only predictor was a partial defect when compared with a circumferential defect ( $54 \%$ vs. $31 \%$ ), which was significant on univariate analysis but not significant on multivariate analysis, as shown in Table 3 . These findings, however, are consistent with similar series in the literature. ${ }^{[4]}$ It is important to note that three quarters of the patients with PCF were managed successfully with conservative management, which suggests that repeated surgical intervention may be ill-advised, especially when poor wound healing is a result of previous radiotherapy.

Table 4: Patient characteristics associated with swallowing dysfunction

\begin{tabular}{|c|c|c|c|c|}
\hline Characteristic & $\begin{array}{c}\text { Without swallowing } \\
\text { dysfunction }\end{array}$ & $\begin{array}{c}\text { With swallowing } \\
\text { dysfunction }\end{array}$ & $\begin{array}{c}P \text { value on univariate } \\
\text { analysis }\end{array}$ & $\begin{array}{c}P \text { value on multivariate } \\
\text { analysis }\end{array}$ \\
\hline \multicolumn{5}{|l|}{ Age (years) } \\
\hline$<60$ & 26 & 9 & 0.617 & - \\
\hline$\geq 60$ & 15 & 7 & & \\
\hline \multicolumn{5}{|l|}{ Sex } \\
\hline Male & 32 & 12 & 0.805 & - \\
\hline Female & 9 & 4 & & \\
\hline \multicolumn{5}{|l|}{ Pharyngeal defect } \\
\hline Circumferential & 23 & 8 & 0.677 & - \\
\hline Partial & 18 & 8 & & \\
\hline \multicolumn{5}{|l|}{ Site } \\
\hline Pharyngeal & 21 & 13 & $0.046^{*}$ & $0.003^{*}$ \\
\hline Laryngeal & 19 & 3 & & \\
\hline \multicolumn{5}{|l|}{ Flap used } \\
\hline Regional & 30 & 12 & 0.413 & - \\
\hline Free & 9 & 6 & & \\
\hline
\end{tabular}

${ }^{*}$ Statistically significant 
Our surgical technique of closure was standardised for all cases. We utilised interrupted suturing in two layers with 3-0 vicryl. Proximally, the flap was spatulated, while distally a lock-and-key figure was used for approximation. The ideal technique of closure and the resultant fistula rates have been intensely debated. One of the first articles to address this was by $\mathrm{Su}$ and Chiang ${ }^{[5]}$ who advocated placement of the T-shaped suture line posteriorly. Cho et al. ${ }^{[6]}$ suggested a modification to the flap design, by overlapping of the vertical suture line with de-epithelialised skin, using a two-layered closure and triangular flaps at the distal anastomotic site (to reduce anastomotic stricture). Although no consensus has been reached, it is important to remember the principles of closure; the approximation needs to be watertight with just adequate approximation so as to not compromise the vascularity of the suture line. In addition, in cases with extensive defects where fistula is likely, it is important to isolate the carotid vessels from the repair and potential area of leak to prevent life-threatening haemorrhage.

The choice of flap for reconstruction is often determined by the extent of the defect, technical expertise and patient fitness. Our institutional protocol was as discussed earlier; however, modifications are needed according to patient fitness and body habitus. For patients unfit for prolonged anaesthesia, regional flaps are used. The bulk of individual flaps needs to be assessed on a patient before taking a decision on choice of flap.

Swallowing dysfunction was another important end-point of our study. A successful pharyngeal reconstruction entails complete oral alimentation without dysphagia. At last follow-up, $72 \%$ of our cohort was on full oral alimentation, with $40 \%$ achieving this within the first 3 months following surgery. Videofluoroscopic assessments are crucial in these patients; it is estimated that up to $50 \%$ of patients suffer from dysphagia following laryngectomy that adversely impacts their quality of life.$^{[4]}$ Continuous assessment by a swallowing therapist leads to early identification and treatment in many of these cases. In our cohort, patients with hypopharyngeal disease had a significantly higher incidence of dysphagia. This is understandable as flap reconstructions do not contribute to propulsion of the bolus and the loss of intrinsic pharyngeal mucosa is likely to correlate with poor propulsion in the neopharynx..$^{[7]}$
Speech rehabilitation with tracheo-oesophageal puncture and prosthesis insertion was done in a little over a third of patients, with a majority of them functioning well. In gastric pull-up and jejunal flaps, the puncture has been shown to be successful, but the voice quality is often poor with a gurgling quality when compared to skin-lined flaps. ${ }^{[6]}$ The decision of primary versus secondary tracheo-oesophageal is controversial, however, if the patient is likely to have a PCF, the puncture is deferred till complete healing. For patients in whom prosthesis placement is unsuccessful or ill-advised, electrolarynx remains a viable method of speech rehabilitation.

\section{CONCLUSION}

Pharyngeal reconstruction following TL is feasible with good results. Majority of the patients, who swallow, regain acceptable swallowing function within 3 months. Most of the early post-operative fistulae can be managed conservatively. The incidence of stricture formation is low, and these are often amenable to dilatation. Patients with pharyngeal disease have poorer swallowing outcomes and higher rates of fistula.

\section{Financial support and sponsorship}

Nil.

\section{Conflicts of interest}

There are no conflicts of interest.

\section{REFERENCES}

1. Patel UA, Keni SP. Pectoralis myofascial flap during salvage laryngectomy prevents pharyngocutaneous fistula. Otolaryngol Head Neck Surg 2009;141:190-5.

2. Lewin JS, Barringer DA, May AH, Gillenwater AM, Arnold KA, Roberts DB, et al. Functional outcomes after circumferential pharyngoesophageal reconstruction. Laryngoscope 2005;115:1266-71.

3. Ganly I, Patel S, Matsuo J, Singh B, Kraus D, Boyle J, et al. Postoperative complications of salvage total laryngectomy. Cancer 2005;103:2073-81.

4. Clark JR, Gilbert R, Irish J, Brown D, Neligan P, Gullane PJ, et al. Morbidity after flap reconstruction of hypopharyngeal defects. Laryngoscope 2006;116:173-81.

5. Su CY, Chiang YC. The fabricated radial forearm flap in pharyngolaryngeal surgery: Saliva leakage and its prevention. Br J Plast Surg 1995;48:212-7.

6. Cho BC, Kim M, Lee JH, Byun JS, Park JS, Baik BS, et al. Pharyngoesophageal reconstruction with a tubed-free radial forearm flap. J Reconstr Microsurg 1998;14:535-40.

7. Walther EK, Herberhold C. Computerized manometry and deglutition after pharyngolaryngeal tumor resections. Laryngorhinootologie 1993;72:67-72. 